\title{
Nano-Structuring by Molecular Self-Assembly
}

\author{
Caterina Minelli, Nicolas Blondiaux, Myriam Losson, Martha Liley, Sylvia Jeney, \\ Christian Hinderling, Raphaël Pugin, Derk Joestera, François Diedericha, Julius Vancsob, \\ Mark Hempenius ${ }^{b}$, and Harry Heinzelmann*
}

\begin{abstract}
In contrast to conventional lithography techniques which will soon hit their limits in terms of feature size and fabrication cost, recent years have seen considerable progress in the development of selfassembling nano-structured surfaces. In the work reported here, surface structures on the submicron scale have been created from polymer, copolymer, nanoparticle, and dendrimer building blocks by employing phenomena such as self-assembly, self-organization and non-equilibrium processes. This low-cost approach is expected to result in a new generation of surfaces with novel physical and chemical properties.
\end{abstract}

Keywords: Dendrimer $\cdot$ Nanoparticle $\cdot$ Nanostructures $\cdot$ Polymer $\cdot$ Self-Assembly

\section{Introduction}

In nanotechnology, the fabrication of surfaces and thin films with controlled nanometer features remains an active area of research. The rational design of complex nanoarchitectures with anticipated novel physical properties is not merely of theoretical interest but also has potential applications in many existing and emerging fields

${ }^{*}$ Correspondence: Dr. H. Heinzelmann CSEM S.A.

Nanoscale Technolology and Biochemical Sensing Jaquet-Droz 1

CH-2007 Neuchâte

Tel.: +4132 7205533

Fax: +4132720 5750

E-Mail: harry.heinzelmann@csem.ch

aOrganic Chemistry Laboratory

ETH Zurich

ETH-Hönggerberg

$\mathrm{CH}-8093$ Zurich

bScience and Technology Department

University of Twente

PO Box 217 7500AE Enschede, Netherlands such as material science, optics, electronics and biotechnology.

Indeed, if manipulation on the nanoscale is a route to the miniaturization of functional micro-devices, controlled surface nano-patterning can also provide systems with new chemical or physical properties: properties that rely on physical principles that become dominant only at the macro or microscopic level. This can lead to a number of interesting effects, with industrial and technical utility. Among the physical properties that can be influenced by nanostructuring are: electronic properties, wettability, friction, reflectivity, and fluid dynamics. At these scales the chemical behavior of materials depends on the structure; even gold loses its chemical inertness and starts to show exciting catalytic properties if it is divided into particles of nanometer dimensions [1]. Surface nano-structures are also attracting attention in biotechnology where they are expected to find application in the orientation of biomolecules, cell adhesion, and adsorption. Methods that allow the efficient creation of nano-structures on surfaces of macroscopic sizes at a reasonable cost are therefore highly industrially relevant.

In this context, the goal of the studies carried out at CSEM is to find innovative, flexible and low-cost methods to fabricate nano-structured surfaces on macroscopic substrates using polymeric, copolymeric, and dendrimeric materials and to examine the new physical, chemical, or biological properties of surfaces structured using these methods. There is a special emphasis on structuring mechanisms relying on selfassembly, self-organization, and non-equilibrium processes, i.e. parallel approaches to the fabrication of large-area structures with high short-range order and some long-range order. In addition to this purely bottom-up approach based on self-organization of molecules, we also combine these selforganization methods with top-down, soft lithography techniques such as microfluidics for larger scale patterning of surfaces.

Here we describe some different approaches to structuring surfaces. The first approach uses the ability of a spin-coated solution of two different homopolymers to demix and form micro- to nano-structured thin films with different morphologies. A very similar approach, used to create even smaller structures, with a higher degree of order is based on the phase separation of block-copolymers. This method gives chemically distinct domains with sizes ranging from $10 \mathrm{~nm}$ to $100 \mathrm{~nm}$. Combining homopolymer and copolymer phase separation even allows the creation of polymeric films with structures on two length scales. The final example described here demonstrates the self-assembly and complexation properties of dendrimers, highly branched monodisperse macromolecules with welldefined structure, geometry, and monodispersity. 


\section{Results and Discussion}

\section{Polymer Demixing}

Polymer demixing, as a technique for structuring solid surfaces has several attractive features: firstly, a wide range of very different surface structures can be formed; structures can be produced in both hard (silicon, glass) and soft (polymer) materials; the method is rapid, simple, and compatible with clean-room technologies; non-planar surfaces can be structured. Taken together, these factors make this approach to surface structuring highly relevant for industrial applications.

Structured thin polymer films can be made by casting or spin-coating from a solution of two (or more) immiscible homopolymers. As the solvent evaporates, a threshold concentration is reached, at which point the solution separates into two different phases, each enriched in one of the polymers. Evaporation of the solvent continues, solidifying the polymer film, and 'freezing in' the microphase structure of the film [2]. The structures obtained by this method are stochastic in nature (see Fig. 1 for a typical example), but with characteristic length scales (structure size and separation) that can be controlled by varying such parameters as solvent, polymer size, concentration, temperature, and substrate chemistry. In addition, selective solvents may be used after deposition of the film to remove one or the other of the polymer phases.

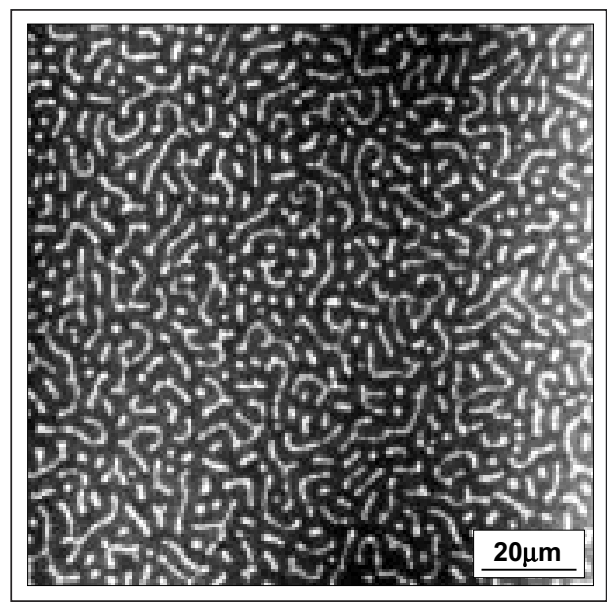

Fig. 1. An optical image of a demixed PS/PMMA film

The influence of the surface energy of the substrate on the microphase structure can be observed in Fig. 2. This figure shows an AFM image of a structured polystyrene (PS)/polymethylmethacrylate (PMMA) film on a gold surface from which the PS has been removed. Before formation of the film, microcontact printing was used to create a striped pattern of two thiols, one very hydrophilic (mercaptohexadecanoic acid) and one hydrophobic (hexadecanethiol), with a periodicity of $2 \mu \mathrm{m}$. On the right of the image, we have the microphase structure of the film on a plain hydrophilic surface: the PMMA phase covers the whole of the surface in a thin film, from which micrometer-sized columns project. The left hand side of the image is the structure formed on the striped pattern: a raised PMMA matrix is observed, with deep holes (corresponding to the PS domains in the original microphase structure) aligned in regular straight lines. Optimization of the film preparation parameters results in a microphase structure that closely follows the patterns on the gold surface.

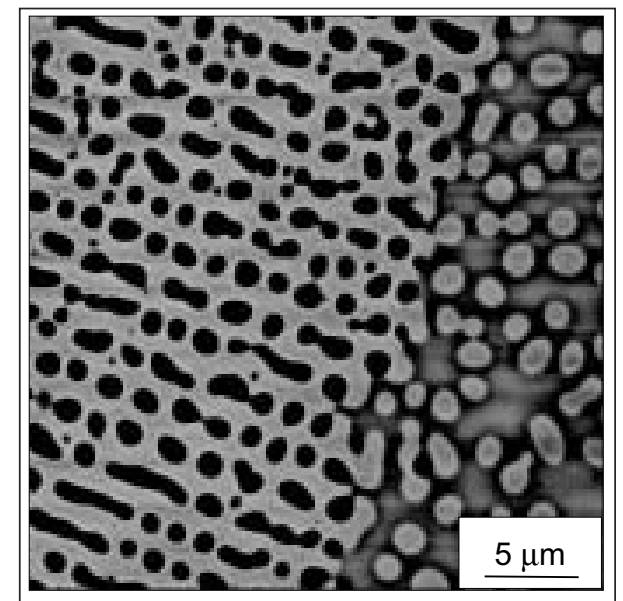

Fig. 2. AFM image of a demixed PS/PMMA film on a patterned gold substrate, after removal of the PS phase. The right hand side of the layer is on a uniform hydrophilic substrate, while the left of the layer is on a region with stripes of alternating hydrophilicity and hydrophobicity.

This technology, initially developed using systems based on organic solvents is now being extended to aqueous systems using water-soluble polymers such as polyvinyl alcohol, polyethylene glycol, and dextran [3]. The elimination of organic solvents allows more environmentally friendly production methods as well as opening new possibilities for applications in fields such as biology: here, structured surfaces of water-soluble polymers are expected to have interesting cell-adhesion properties. The same range of structure form and size (from few microns to tens of nanometers) is available in water-soluble polymers (Fig. 3) as in organic solvent systems. Polymer demixing may also be used to organize objects such as nanoparticles within thin films, based on the different affinities of the objects for the different polymer phases. Working in collaboration with the group of Prof. H. Vogel at the EPF Lausanne, we have used this effect to organize fluorescent CdSe nanoparticles in films of PS/PMMA and have observed a strong partitioning of the particles into the PS phase. Confocal microscopy has been used to study samples with very low concentrations of the nanoparticles, as shown in Fig. 4a and 4b. In these images, we observe a demixed PS/ PMMA film: the higher fluorescence of the PS phase means that the microphase structure of the film is easily distinguishable in the image. There are a number of brighter spots superimposed on the microphase structure, which are the individual fluorescent nanoparticles. The nanoparticles can be identified as single, individual particles, based on their fluorescence behavior (blinking, bleaching, polarization) and are clearly preferentially located within the PS phase of the film. Pre-structuring the substrate by microcontact printing before casting the polymer film, as described above, allows us to arrange the nanoparticles in stripes (Fig. 4c), dots, or other patterns. Further work on this subject will concentrate on quantifying the partitioning of the particles, studying their optical properties in the different polymer phases, and organizing the particles in nanometer scale structures.

\section{Copolymer Lithography}

In order to create even smaller structures, we have investigated the use of the self-organization properties of block-copolymers. If such polymers contain two blocks, which are mutually insoluble, they phase-separate and form chemically distinct domains. The domains formed are well ordered and very small; they are directly related to the size of the polymer chains and are therefore of the order of 10 to $100 \mathrm{~nm}$. Depending on the relative length of the blocks, different morphologies of the domains form e.g. spheres, cylinders, or layers (Fig. 5).Thin films of block-copolymers applied to surfaces thus result in surface patterning with well-ordered chemically distinct phases or domains. The phases can then further be treated with selective reagents, e.g. by doping with metal ions or selectively removing one block with a specific reagent. This was first demonstrated by Mansky et al. [4] using polystyrenebutadiene and polystyrene-isoprene blockcopolymers and ozone to selectively remove the butadiene or isoprene blocks. Park et al. [5] have reported on the generation of $10^{11}$ features $/ \mathrm{cm}^{2}$.

In collaboration with the group of Prof. Vancso at the University of Twente, we are investigating the use of block-copolymers that contain poly(ferrocenyl-dimethylsilane) (PFS) as one constituent (Fig. 6).

This very interesting system was pioneered by Manners and co-workers [6] and further developed in the group of Vancso 

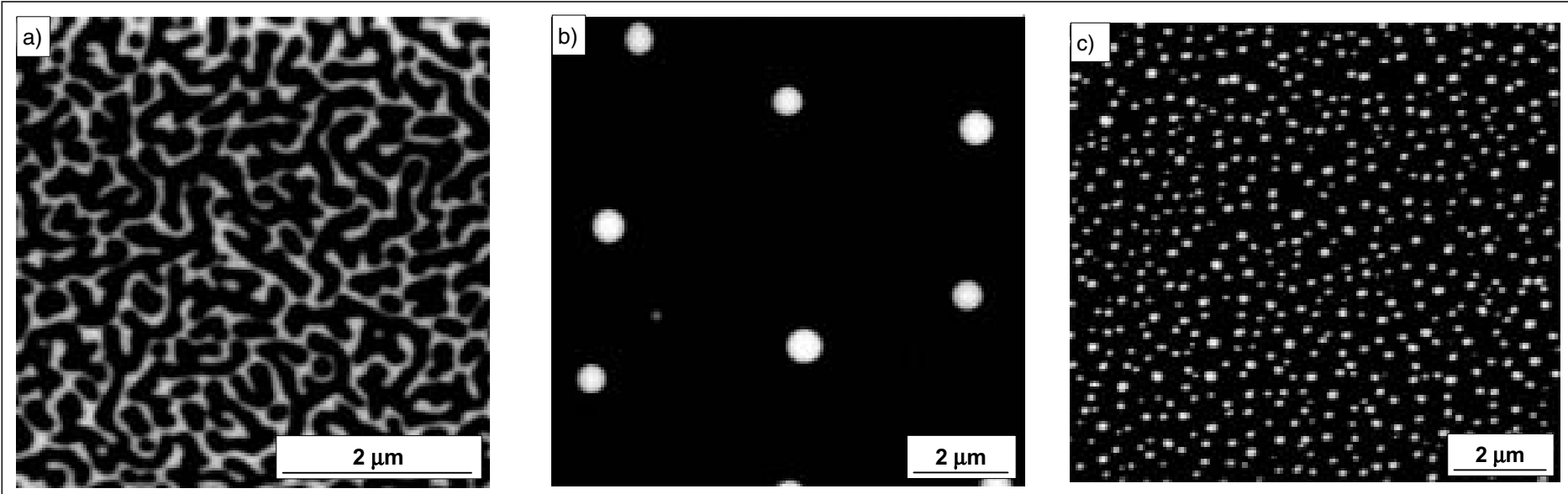

Fig. 3. AFM images of thin films of water-soluble polymers. a) a PVA film after removal of the polyDADMAC phase; b) and c) dextran films after removal of PEG phases

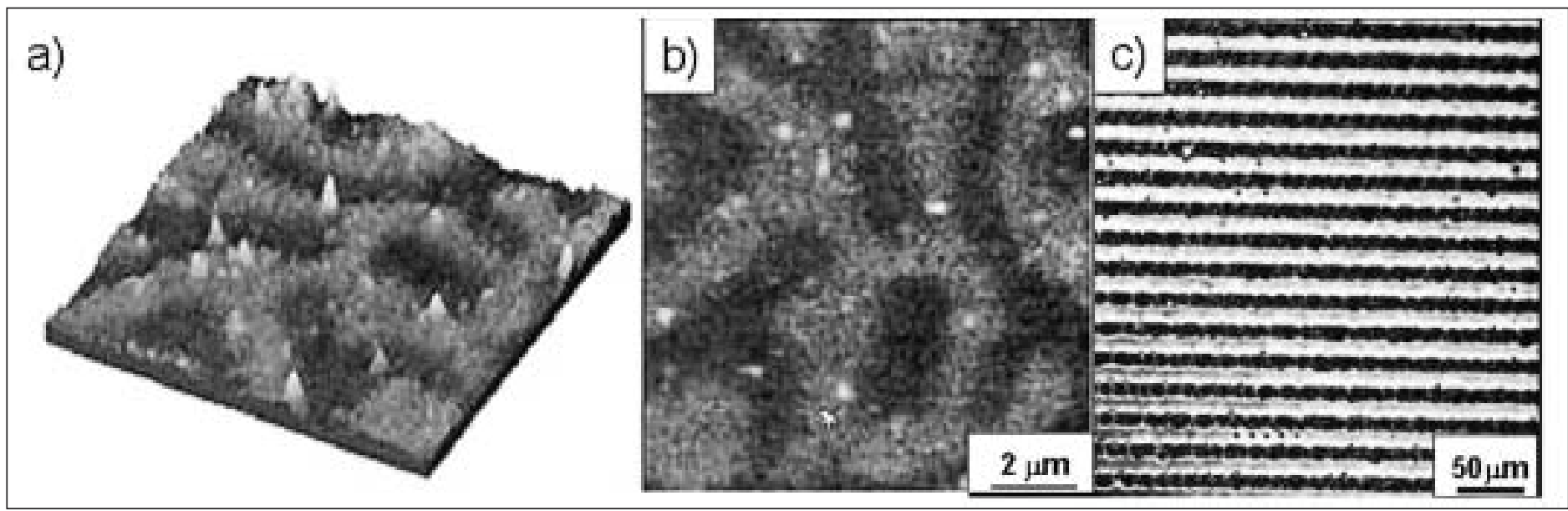

Fig. 4. Fluorescent images of CdSe NPs in PS/PMMA films
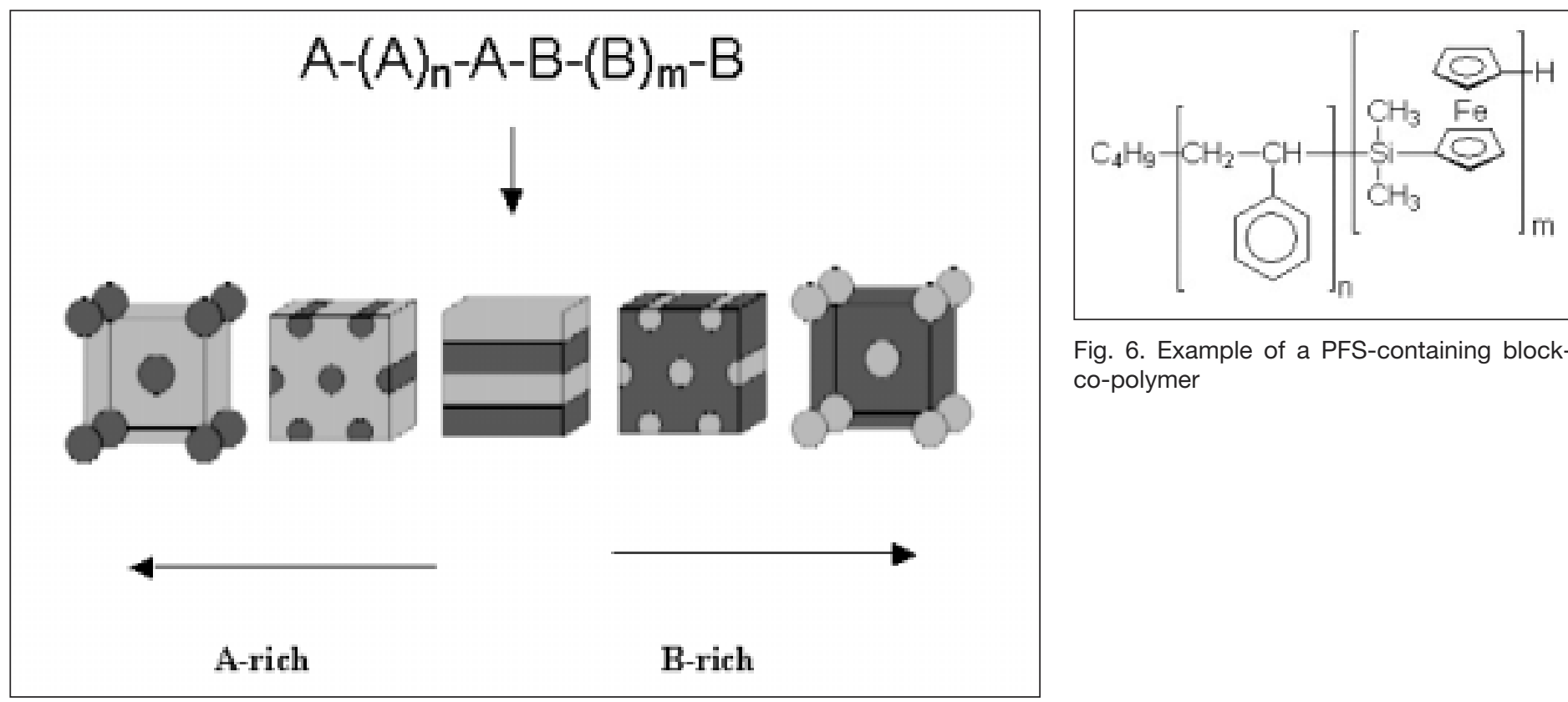

Fig. 6. Example of a PFS-containing blockco-polymer

Fig. 5. Examples of typical self-organized phase-separation patterns. A block-copolymer consisting of monomers $A$ and $B$ undergoes microphase separation. The type of pattern obtained depends on the block-copolymer composition (A/B ratio) 
[7]. The outstanding feature of those systems is the high resistance of PFS to reactive ion etching. Surfaces can thus be structured simply by spin-coating them with a thin block-copolymer film followed by reactive ion etching. This treatment etches away all the polymer except the PFS-containing domains, which are protected by an oxide layer and thus form an effective etch-mask.

This makes the systems well suited for the efficient nano-structuring of surfaces as industrially important as silicon or silicon nitride (Fig. 7).

Combining the two previously discussed methods by using a mixture of a block-copolymer with an incompatible homopolymer can lead to nano-structures within microstructures. This opens the way to structure surfaces on different length scales relaying on self-organization only (Fig. 8).

\section{Dendrimeric Patterned Surfaces}

In order to extend our research to other macromolecules, the last part of this work concerned the use of highly branched dendrimers for the functionalization and structuring of surfaces. Monolayers prepared by the direct adsorption of several kinds of dendrimers onto different substrates have already attracted much attention [8-10]. First, they are highly stable in most cases since dendrimers molecules are strongly adsorbed to the initial surface in contrast to the monopodal attachment of linear alkylbased self-assembled monolayers. Moreover, since such molecules contain a large number of peripheral reactive groups as well as a structure that is molecularly controllable (type of core, the choice of branch point, and distance between successive branch junctions), they turned out to be very efficient in influencing the self-assembly properties of biomolecules [11] such as antibodies or plasmids.

In this context, our first experiments have consisted in patterning surfaces with highly branched PAMAM dendrimers of the fourth generation (ethylenediamine core; 64 terminal $-\mathrm{NH}_{2}$ groups). The ability of such dendrimeric patterned surface to be used subsequently as chemically sensitive interfaces for biomolecules complexation will also be described.

For patterning, the top-down microfluidic approach was first considered to guide the self-assembling of PAMAM onto well-defined areas. PAMAM solution was allowed to flow through 2 and $20 \mu \mathrm{m}$ periodic PDMS channels driven by capillary forces, on photoactive Optodex ${ }^{\circledR}$ coated glass substrate (Fig. 9). After photo-immobilization of dendrimers, the surface was

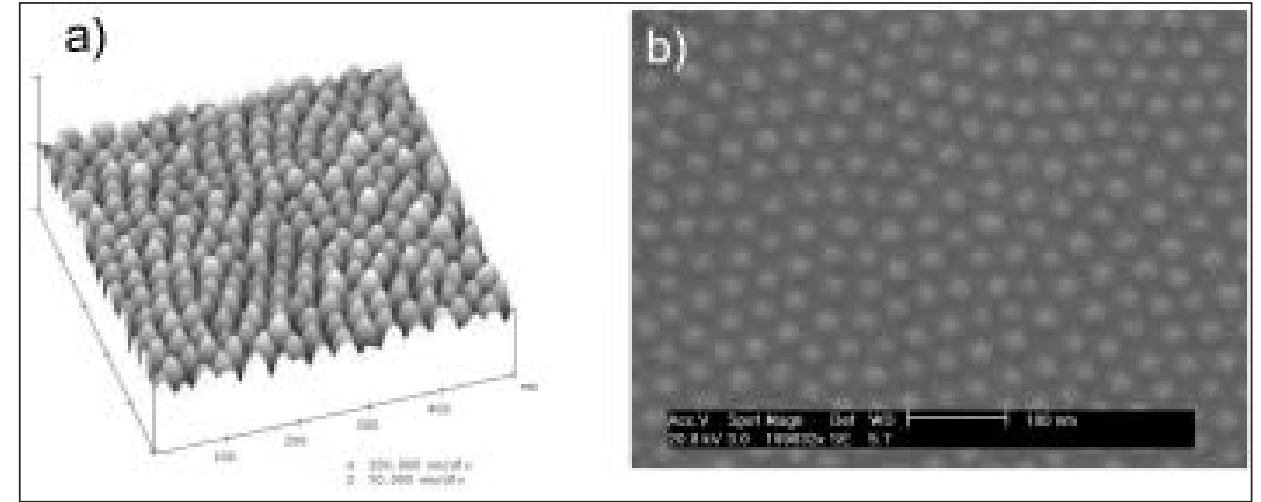

Fig. 7. Tapping mode AFM image (a) and scanning electron microscope (b) images of spin-cast films of polystyrene-poly(ferrocenyldimethylsilane) block-copolymer on a silicon surface after removal of the polystyrene block by $\mathrm{O}_{2}$-reactive ion etching, showing self-organized $30 \mathrm{~nm}$ etch-resistant dots and mid-range ordering

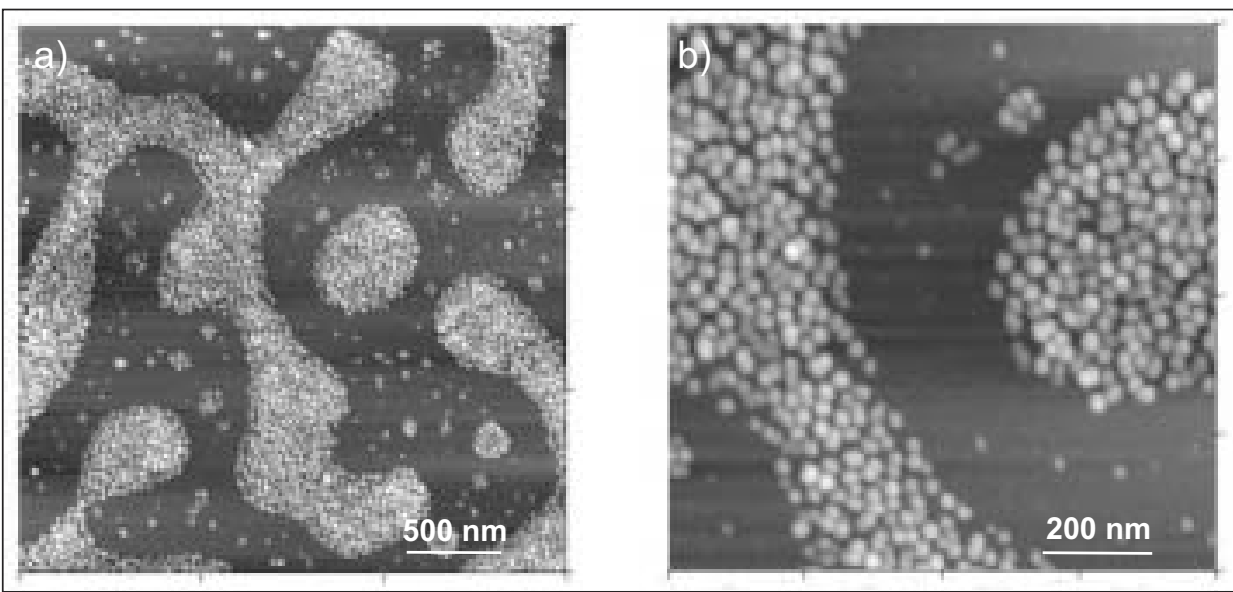

Fig. 8. a) Tapping-mode AFM images of spin cast films of a blend of a polystyrene-poly(ferrocenyl-dimetylsilane) block-copolymer with PMMA after $\mathrm{O}_{2}$-plasma etching showing self organized nano-structures within microstructures. b) zoom of (a)

washed in order to remove non-covalently bound dendrimer molecules and analyzed by atomic force microscopy. The observation of dendrimer stripes with height from 3 to $4 \mathrm{~nm}$ is perfectly in agreement with the $4.5 \mathrm{~nm}$ theoretical diameter of G4-PAMAM and allowed confirmation of the monolayer character of adsorbed dendrimers.

The second step in our study has then consisted of an evaluation of the reactivity of the free side of adsorbed dendritic mole-

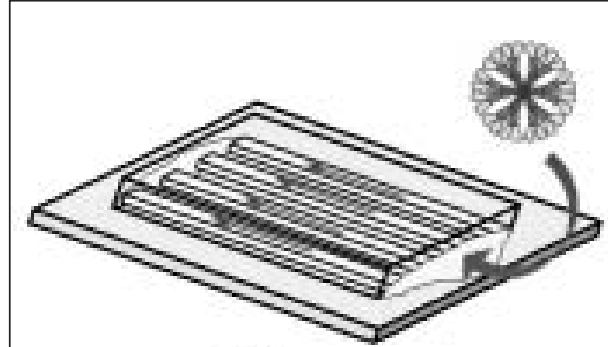

(a) cules. Among all possibilities, we wished to investigate the ability of these pre-functionalized surfaces to coordinate biomolecules.

Dendrimeric patterned surfaces were first tested in complexing fluorescent $\mathrm{IgG}$ antibodies via EDC chemistry. The functionalized substrates were simply dipped in $\mathrm{IgG/NHS/EDC} \mathrm{solution,} \mathrm{washed,} \mathrm{and} \mathrm{ana-}$ lyzed by fluorescence microscopy. Resulting fluorescence images showed regular and homogeneous $\mathrm{IgG}$ architectures with 2
Fig. 9. PAMAM solution sucked by capillary action through the channels of 2 and $20 \mu \mathrm{m}$ periodic PDMS stamps 


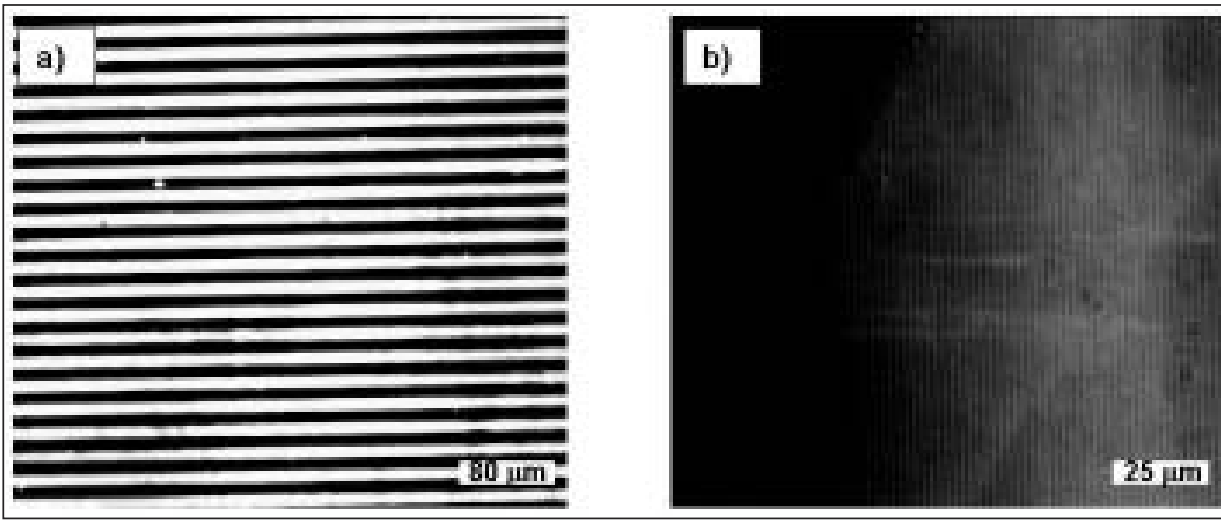

Fig. 10. Fluorescence microscope images of IgG microstructures. PAMAM dendrimer deposited by fluidics through $20 \mu \mathrm{m}$ (a) and $2 \mu \mathrm{m}$ (b) periodic PDMS channels followed by covalent fixation of IgG.

and $20 \mu \mathrm{m}$ periodicities (Fig. 10). These results clearly demonstrated the selective immobilization of IgG by the prefunctionalized dendrimeric surface while non-patterned areas stay unreactive.

Since the efficiency of biomolecules complexation was successfully achieved at a microscopic scale with patterned PAMAM surfaces, our research was recently extended to nano-structured dendrimeric surfaces.

In collaboration with Prof. Diederich from ETH Zürich, nano-structured surfaces were recently obtained using specially designed amphiphilic dendrimers and Langmuir-Blodgett techniques. The ability of dendrimeric surfaces to bind strongly and influence DNA conformation and packing is currently under investigation. First results are very promising and could be related to the recently reported potential of such dendrimers to behave as efficient self-assembling vectors to promote gene delivery therapy [12].

\section{Conclusions}

To sum up, polymer self-assembly and self-organization is a powerful and lowcost approach to structuring surfaces on scales from several microns to a few nanometers. Both hard and soft surface structures can be produced, and the sizes and shapes of the structures can be tuned as required. Nanoparticles and macromolecules such as DNA can be complexed and organized on the surface.

The techniques described briefly in this article are now being developed for applications in widely different fields. To mention just a few: nanoporous polymer films are being developed for use in photo-quality printer paper; silicon surfaces will be nanostructured to modify the mechanical properties of microsystems; magnetic nano- particles are being organized into sub-micron patterns with a view to memory storage applications; structured hydrogel films are being explored for uses in biosensors.

\section{Acknowledgements}

The work reviewed in this paper was partly funded by the EU project Polynano, TopNano 21, the Swiss National Fonds and the National Center of Competences in Research (NCCR) in Nanosciences. We thank them for their support.

We also gratefully thank Dr. M. Hempenius and I. Korczagin (University of Twente) for providing PFS diblock-copolymers and helpful discussions as well as I. Geissbühler and Prof. H. Vogel (EPF-Lausanne) for providing the CdSe nanoparticles.

Received: July 14, 2003

[1] A.T. Bell, Science 2003, 299, 1688.

[2] S. Walheim, M. Boltau, J. Mlynek, G. Krausch, U. Steiner, Macromolecules 1997, 30, 4995.

[3] I. Hopkinson, M. Myatt, Macromolecules 2002, 35, 5153.

[4] P. Mansky, C.K. Harrison, P.M. Chaikin, R.A. Register, N. Yao, Appl. Phys. Lett. 1996, 68, 2586; US Pat. US5948470

[5] M. Park, C. Harrison, P.M. Chaikin, R.A. Register, D.H. Adamson, Science 1997, 276, 1401.

[6] Y. Ni, R. Rulkens, I. Manners, J. Am. Chem. Soc. 1996, 118, 4102.

[7] a) R.G.H. Lammertink, M.A. Hempenius, J.E. Van den Enk, V.Z.-H. Chan, E.L. Thomas, G.J. Vancso, Adv. Mater. 2000, 12, 98; b) R.G.H. Lammertink, D.J. Versteeg, M.A. Hempenius, G.J. Vancso, $J$. Polym. Sci. A: Polym. Chem. 1998, 36, 2147; c) R.G.H. Lammertink, E.L. Thomas, M.A. Hempenius, G.J. Vancso, $J$. Polym. Sci. B: Polym. Phys. 1999, 37, 1009.

[8] A. Hierlemann, J.K. Campbell, L.A. Baker, R.M. Crooks, A.J. Ricco, J. Am. Chem. Soc. 1998, 120, 5323.

[9] A. Friggeri, H. Schönherr, H.-J. van Manen, B.-H. Huisman, G.J. Vancso, J. Huskens, F.C.J.M. van Veggel, D. Reinhoudt, Langmuir 2000, 16, 7757.
[10] D.C. Tully, K.Wilder, J.M.J. Fréchet, A.R. Trimble, C.F. Quate, Adv. Mater. 1999, 11, 314.

[11] H.C. Yoon, M.Y. Hong, H.S. Kim, Anal. Biochem. 2000, 282,121.

[12] D. Joester, M. Losson, R. Pugin, H. Heinzelmann, E. Walter, H.-P. Merkle, F. Diederich, Angew. Chem. Int. Ed. 2003, 42, 1486. 